Research Paper

\title{
Bmil Drives Stem-Like Properties and is Associated with Migration, Invasion, and Poor Prognosis in Tongue Squamous Cell Carcinoma
}

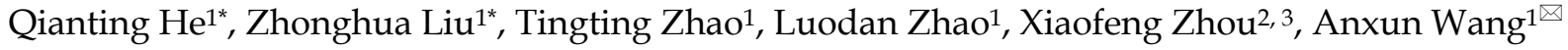 \\ 1. Department of Oral and Maxillofacial Surgery, First Affiliated Hospital, Sun Yat-Sen University, Guangzhou, 510080, China \\ 2. Center for Molecular Biology of Oral Diseases, College of Dentistry, University of Illinois at Chicago, Chicago, IL, 60612-7213, USA. \\ 3. Department of Periodontics, College of Dentistry, University of Illinois at Chicago, Chicago, IL, 60612-7213, USA. \\ *These authors contributed equally to this work.
}

$\triangle$ Corresponding author: Anxun Wang, Department of Oral and Maxillofacial Surgery, First Affiliated Hospital, Sun Yat-Sen University. 58 Zhongshan Road II, Guangzhou, 510080, P.R.China; Phone: +86-0-13724896216; E-mail: anxunwang@yahoo.com.

( ) Ivyspring International Publisher. This is an open-access article distributed under the terms of the Creative Commons License (http://creativecommons.org/ licenses/by-nc-nd/3.0/). Reproduction is permitted for personal, noncommercial use, provided that the article is in whole, unmodified, and properly cited.

Received: 2014.08.25; Accepted: 2014.10.17; Published: 2015.01.01

\begin{abstract}
Bmil (B-cell-specific Moloney murine leukemia virus insertion site 1) had been found to involve in self -renewal of stem cells and tumorigenesis in various malignancies. The purpose of this study is to evaluate the role of Bmil in the development of tongue squamous cell carcinoma (TSCC) and its functional effect on the migration and invasion of TSCC. Initially, immunohistochemistry revealed that Bmil overexpression was a common event in premalignant dysplasia, primary TSCC, and lymph node metastases and was associated with a poor prognosis. A significant correlation between Bmil and SOD2 (manganese superoxide dismutase) expression was observed. Side population (SP) cells were used as cancer stem-like cells and further assessed by sphere and colony formation assays, and the expression of stem cell markers. TSCC cells with higher migration and invasion ability (UMI cell lines) showed a higher proportion of SP cells and Bmil expression than TSCC cells with lower migration and invasion ability (UM2 cell lines). Knockdown of Bmil in UM1 or SP cells inhibited migration and invasion and decreased the sphere and colony formation, and the expression of stem cell markers and SOD2. Direct binding of C-myc to the Bmil promoter was demonstrated by chromatin immunoprecipitation and luciferase assays. Moreover, C-myc knockdown in SP cells inhibited their migration and invasion and decreased the expression of Bmil and SOD2. Our results indicate that the deregulation of $\mathrm{Bmil}$ expression is a frequent event during the progression of TSCC and may have a prognostic value for patients with this disease. The Bmil-mediated migration and invasion of TSCC is related to cancer stem-like cells and involves the C-myc-Bmil-SOD2 pathway.
\end{abstract}

Key words: Tongue squamous cell carcinoma; Cancer stem-like cell; migration; invasion; prognosis; Bmi1.

\section{Introduction}

As a type of common malignant tumor in the oral cavity, tongue squamous cell carcinoma (TSCC) has a high mortality rate due to early metastasis and recurrence. Although the level of healthcare has greatly improved, the cure rate of TSCC remains unsatisfactory, and the 5-year survival rate is $50 \%[1]$.
One of the reasons for treatment failure is thought to be related to the presence of a subpopulation of cells within the tumor called cancer stem cells (CSCs). In head and neck squamous cell carcinomas (HNSCCs), including TSCC [2], CSCs have been shown to have an integral role in tumor initiation, disease progression, 
metastasis, and treatment resistance [3-6].

B-cell-specific Moloney murine leukemia virus insertion site 1 (Bmi1) is a member of the Polycomb group of chromatin-modifier proteins. As a stem cell marker, Bmi1 plays a key role in the functioning of endogenous stem cells and CSCs [7,8]. Moreover, Bmil had been found to be related with many other cancer stem cell markers [9-12], such as ALDH1, CD44, Oct4, SOX2, Nanog and ABCG2. Tsai et al found that Sphere-forming/self-renewal capability was increased in cisplatin-resistant OC2 cells (oral squamous carcinoma cells). Cisplatin-resistant OC2 cells highly expressed the stemness markers (Nanog, Oct4, Bmi1, CD117, CD133, and ABCG2) and increased migration/invasion/clonogenicity ability [9]. Seo et al also found a connection between BMI-1 and Sox2 in maintaining self-renewal and identified BMI-1 as a key mediator of Sox2 function [10]. In human cancers, Bmil overexpression had been found to drive stem-like properties associated with the induction of the epithelial-mesenchymal transition that promotes invasion, metastasis, and poor prognosis [13]. Indeed, many studies have reported that Bmil is overexpressed in HNSCC cells when compared with normal epithelium and is thought to influence cell proliferation and survival in HNSCC [13-16]. In contrast, Hayry`s study found that Bmi-1-negative tumors showed a correlation with a poor prognosis in TSCC [17]. Thus, more evidence is needed to confirm the relationship between Bmil and the development of TSCC.

Although Bmi1 has been found to be associated with HNSCC $[10,11]$, the mechanistic rationale for an increased metastatic capacity of tumor cells overexpressing Bmi1 is still ambiguous and requires further investigation. As an oncogenic transcription factor, the C-myc protein recognizes an E-box recognition site in the promoter of its target genes and then exerts a wide array of biological functions in different cellular models, including cell cycle control, cell differentiation, and metastasis [18-20]. C-myc has been documented to regulate the expression of an unusually large number of target genes, including Bmi1 in nasopharyngeal carcinoma [21]. In our previous studies [22-24], we identified that the manganese superoxide dismutase (SOD2)-dependent production of $\mathrm{H}_{2} \mathrm{O}_{2}$ contributes to the migration and invasion of TSCC via the Snail (Snai1 and Slug) signaling pathway. However, the relationship between Bmi1 and SOD2 has not been uncovered to date.

The goal of this study was to investigate the role of Bmi1 in the development of TSCC and its mechanism in the migration and invasion of TSCC. First, we investigated the role of Bmi1 in the development and progress of TSCC. We then investigated whether the migration and invasion of TSCC mediated by Bmi1 were related to CSCs and whether the C-myc-Bmi1-SOD2 pathway mediates the metastasis of TSCC. Our findings suggest that Bmi1 is an important factor in the development and prognosis of TSCC. In addition, the Bmi1-mediated migration and invasion of TSCC is related to cancer stem-like cells and involves the C-myc-Bmi1-SOD2 pathway.

\section{Methods and materials}

\section{Patients and tissues}

The clinical samples used in this study were obtained from 77 cases of TSCC, 22 cases of premalignant tongue (leukoplakia, LP), and 12 normal tongue biopsies, which had been used in our previous study $[23,25]$. Clinical characterizations of these samples are summarized in Supplementary Material: Table S1. Among the 77 cases of TSCC that we examined, follow-up results were available for 52 cases; the median duration of follow-up was 77 months (range: 8-116 months). Survival was calculated based on the date of surgery and the date of latest follow-up (or death). This study was approved by the ethical committee of the First Affiliated Hospital, Sun Yat-Sen University.

\section{Immunohistochemistry analysis}

The immunohistochemistry analysis was performed as previously described $[25,26]$ using an anti-Bmi1 antibody (Cell Signaling Technology, Beverly, MA, USA). Sample evaluation via immunostaining was performed by 3 independent pathologists blinded for the clinical data according to criteria of staining intensity described previously by Li et al [27]. All areas of tumor cells within each section were analyzed. All tumor cells in ten random high-power fields were counted. A scale of 0 to 3 was used to score the relative intensity, with 0 corresponding to no detectable immunoreactivity, and 1, 2, and 3 equivalent to low, moderate, and high staining, respectively. The representative images of each score (0-3) were supplied in Supplementary Material: Figure S1.

\section{Cell culture and transfection}

Human TSCC cell lines (UM1 and UM2) [24], with UM1 having a higher migration and invasion ability than UM2, were maintained in DMEM/F12 (Gibco, Carlsbad, CA, USA) containing 10\% fetal bovine serum (Hyclone, Logan, Utah, USA), $100 \mathrm{U} / \mathrm{ml}$ penicillin, and $100 \mu \mathrm{g} / \mathrm{ml}$ streptomycin in a $37^{\circ} \mathrm{C}$ incubator with $5 \% \mathrm{CO}_{2}$. For further study, a Bmi1 siRNA, C-myc siRNA, and control non-targeting siRNA (Genepharma, Shanghai, China) were transfected into the TSCC cells using Lipofectamine Transfection Reagent (Invitrogen, Carlsbad, CA, USA) according to the manufacturer's instructions, as pre- 
viously described [28]. Three sequences of Bmi1 or C-myc siRNA were used, and then the sequence that had the best knockdown effect was chosen. The sequences of siRNA used for transfection are shown in Supplementary Material: Table S2.

\section{Cell migration and invasion assay}

The cell migration and invasion assay were performed according to our previous study [24,28]. Briefly, for the migration assays, cells were seeded in the upper chamber of Transwell devices (Corning, Steuben, NY, USA) with a membrane containing 8 - $\mu$ m-diameter pores in serum-free medium. After 24 $h$, the cells on the lower surface of the membrane were fixed and stained with DAPI solution in the dark. Three random fields were captured at $20 \times$ magnification. For the invasion assays, Biocoat Matrigel invasion chamber inserts (BD Biosciences, San Diego, CA, USA) were equilibrated for $2 \mathrm{~h}$ at $37^{\circ} \mathrm{C}$ in serum-free medium. The cells were seeded in the upper chamber and allowed to invade through the Matrigel to the lower chamber for $24 \mathrm{~h}$. After $24 \mathrm{~h}$, the number of invaded cells (lower side of the membrane) was counted as described above.

\section{Western blot analysis}

Western blots were performed as described previously [24] using antibodies specific for Bmi1, SOD2, Slug (Cell Signaling Technology, Beverly, MA, USA), Nanog (Aviva Systems Biology, San Diego, CA, USA), C-myc, ABCG2 (ATP-binding cassette transporters) (Santa Cruz, Dallas, Texas, USA) and GAPDH (Sigma, San Louis, MO, USA). The results obtained from western blot were quantified by Quantity One software (Bio-Rad) and shown in Supplementary Material: Figure S2.

\section{Side population sorting}

The TSCC side population was sorted as previously described [29]. Briefly, TSCC cells were incubated at $37^{\circ} \mathrm{C}$ for 90 minutes in the dark with $5 \mu \mathrm{g} / \mathrm{ml}$ Hoechst 33342 (Sigma, San Louis, MO, USA) and intermittent mixing either alone or in the presence of 50 $\mu \mathrm{M}$ verapamil (Sigma, San Louis, MO, USA), an inhibitor of ABC transporters. The cells were then centrifuged and resuspended in ice-cold HBSS. The samples were sorted using a FACSAria II high-speed cell sorter (BD Biosciences, San Diego, CA, USA). Propidium iodide $(2 \mu \mathrm{g} / \mathrm{ml})$ was added to exclude dead cells. Hoechst 33342 was excited with the UV laser at $350 \mathrm{~nm}$, and the fluorescence emission was measured with 405 / BP30 (Hoechst blue) and 570 / BP20 (Hoechst red) optical filters. After sorting, the SP and non-SP cells were used for further experiments.

\section{Sphere and colony formation assays}

For the spheroid-forming assay, cells were plated in 24-well ultralow attachment plates (Corning, Steuben, NY, USA) at a density of 1000 cells/ $\mathrm{ml}$ in 100 $\mu \mathrm{l}$ of stem cell medium containing DMEM/F12 plus $1 \% \mathrm{~N} 2,10 \mathrm{ng} / \mathrm{ml}$ human recombinant bFGF, 10 $\mathrm{ng} / \mathrm{ml}$ human recombinant EGF, and $1 \%$ antibiotic-antimycotic (Life Technologies, Carlsbad, CA, USA). Sphere formation (20 $\mathrm{mm}$ in diameter) in each well was calculated at days 14 after seeding.

For the colony formation assay, cells were plated at approximately 200 cells per well in 6-well coated plates in DMEM/F-12 supplemented with $10 \%$ FBS. The medium was changed twice a week. After 14 days, the cells were fixed in $4 \%$ formaldehyde and stained with Giemsa. Colonies larger than $1 \mathrm{~mm}(>50$ cells/clone) in diameter were counted.

\section{Chromatin immunoprecipitation (ChIP)}

ChIP assays were performed as described previously [30] following the manufacturer's recommendations (Upstate Biotechnology, Buffalo, NY, USA). A total of $1 \times 107$ UM1 or UM2 cells were crosslinked with formaldehyde, and the cell pellet was lysed and sonicated to shear the DNA into fragments of 200-500 bp. Following precleaning, 1\% of each chromatin supernatant was used as the input loading control; the remaining chromatin supernatant was incubated with C-myc antibodies or preimmune IgG (PI) for immunoprecipitation. Protein-chromatin complexes were eluted and reverse-cross-linked to recover free DNA. Purified DNA was analyzed by a quantitative polymerase chain reaction (PCR) analysis with SYBR Green qPCR SuperMix Kit (Invitrogen, Carlsbad, CA, USA), as previously described [28]. The primers for Bmi1 (NM_005180) were as follows: F, 5' taattcccaggccgecctta 3'; R, 5' caccggctccaaaatggctc 3'.

\section{Dual luciferase reporter assay}

A dual luciferase assay was performed as described previously [28]. Briefly, the dual luciferase reporter gene construct for Bmi1 (pGL-Bmi1) was created by cloning a 925-bp fragment from the promoter of Bmi1 (NM_005180, containing the C-myc binding site (gagcacgtgac)) into the KpnI and HindIII sites of the pGL3-Control firefly luciferase reporter vector (Promega, Madison, WI, USA). The corresponding mutant constructs (pGL-Bmilmt) were created by replacing the C-myc binding site in the promoter of Bmi1 with 5'-ctcgagcactg-3'. A plasmid containing C-myc was also constructed by cloning a 1365-bp fragment of C-myc cDNA (NM_002467.4) into the BamHI and XhoI sites of pcDNA3.1. The constructs were then verified by sequencing. The cells were transiently cotransfected with pGL-Bmi1 and 
pcDNA3-C-myc using Lipofectamine (Life Technologies, Carlsbad, CA, USA). The luciferase activity was measured with a luminometer (Tecan, San Jose, CA, USA), and the activities were normalized based on Renilla activity and the protein concentration (cotansfected with pRL-SV40).

\section{Cell proliferation assays}

Proliferation was measured using a Cell Counting Kit-8 (CCK-8) assay. Briefly, cells were seeded in 96-well plates at a density of $2 \times 103$ cells per well. Cell proliferation was analyzed by incubating the cells with CCK-8 (Dojindo, Kumamoto, Japan) according to the manufacturer's instructions at $72 \mathrm{~h}$ after transfection. Absorbance (A) was measured at $450 \mathrm{~nm}$, and the cell inhibition rate was calculated as $\left(1-\mathrm{A}_{\text {treated }} / \mathrm{A}_{\text {control }}\right) \times 100 \%$.

\section{Statistical analysis}

The results are representative of at least three independent experiments; data are presented as the mean \pm standard error. The data were analyzed using the Statistical Package for the Social Science (SPSS, Chicago, IL, USA), Version 17.0. Pearson and Spearman Correlation Coefficients were used to assess correlations among the gene expression and clinical and histopathological parameters. A one-way ANOVA and Student's t-test were used to compare differences between groups. Survival curves were plotted using the Kaplan-Meier method and compared with the log-rank test. For all statistical analyses, $\mathrm{P}<0.05$ was considered statistically significant.

\section{Results}

\section{Bmil plays an important role in the develop- ment of TSCC and correlates with SOD2}

To confirm the role of Bmi1 in the development of TSCC, the expression of Bmi1 was examined by immunohistochemistry (IHC). As illustrated in Figure 1, Bmi1 IHC staining was observed in the cell nucleus. The percentage of positive cells and the staining intensity in LP and TSCC tissues were increased compared to normal tongue mucosa. Bmi1 expression was significantly higher in LP and TSCC than in normal tongue tissue (Figure 2A) and in moderate and severe LP than in light LP (Figure 2B). Among the TSCC cases, the expression level of Bmi1 was significantly higher in the patients with a positive node metastasis status $(\mathrm{pN}+)$ than those with a negative status $(\mathrm{pN}-)$ (Figure 2C) and in late-stage (stages III and IV) than in early-stage disease (stages I and II) (Figure 2D). No difference in the Bmi1 expression level was observed in TSCC cases with regard to different age, gender, pT stage, and pathological differentiation. As illustrated in Figure 2E, there was a significantly higher 5-year survival rate in the low Bmi1 expression group (mean survival $=105$ months) than in the high Bmi1 expression group (mean survival $=42$ months). The 5-year survival rate, as assessed by the Kaplan-Meier method, was $85.7 \%$ in the low Bmi1 expression group, whereas it was only $25 \%$ in the high Bmi1 expression group.
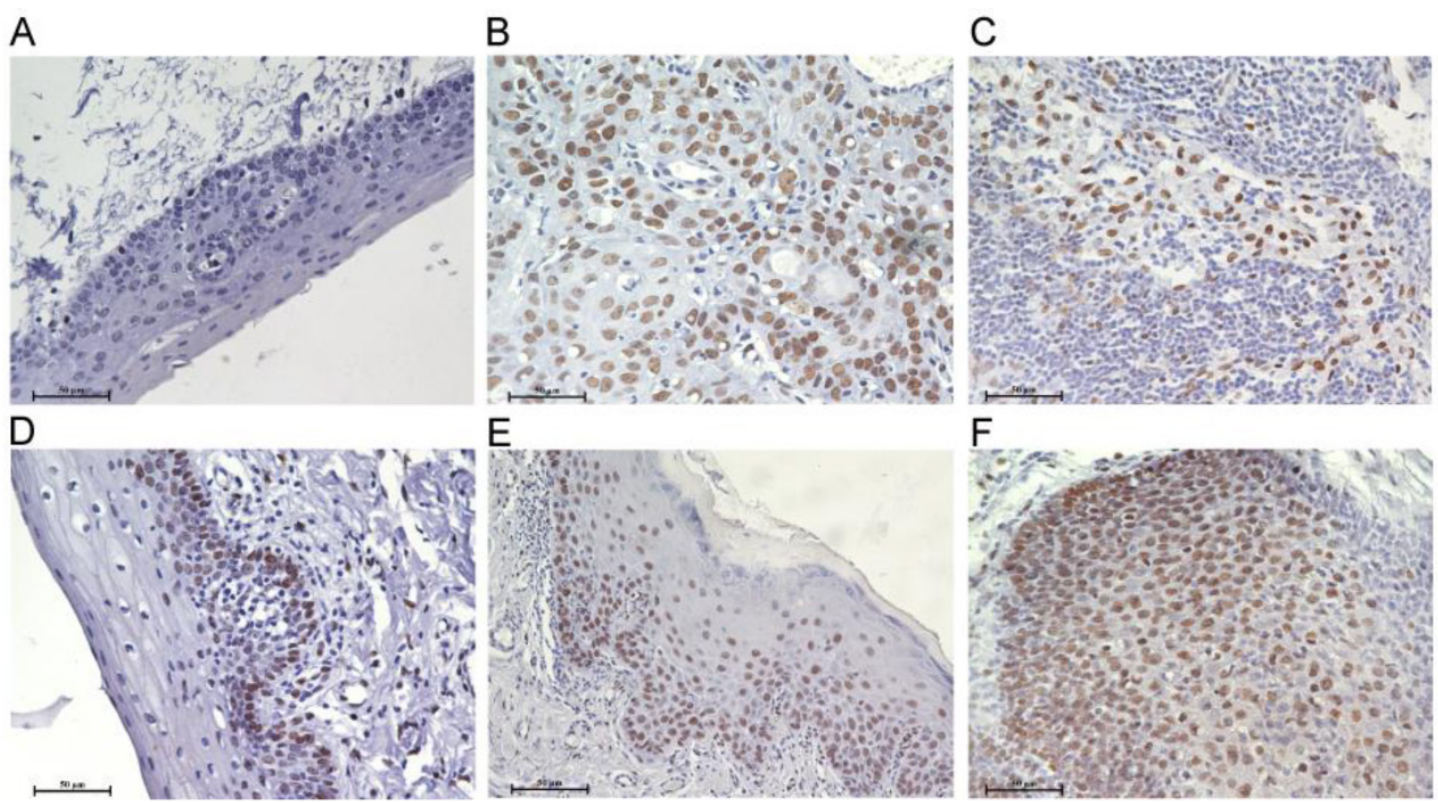

Figure 1. Bmil deregulation in the development of TSCC. Immunohistochemistry analyses for Bmil were performed as described in Material and Methods. A: normal tongue mucosa, B: TSCC, C: lymph node metastasis tissue of TSCC, D: light LP, E: moderate LP, F: severe LP. (Scale bar: $50 \mu \mathrm{m})$. 

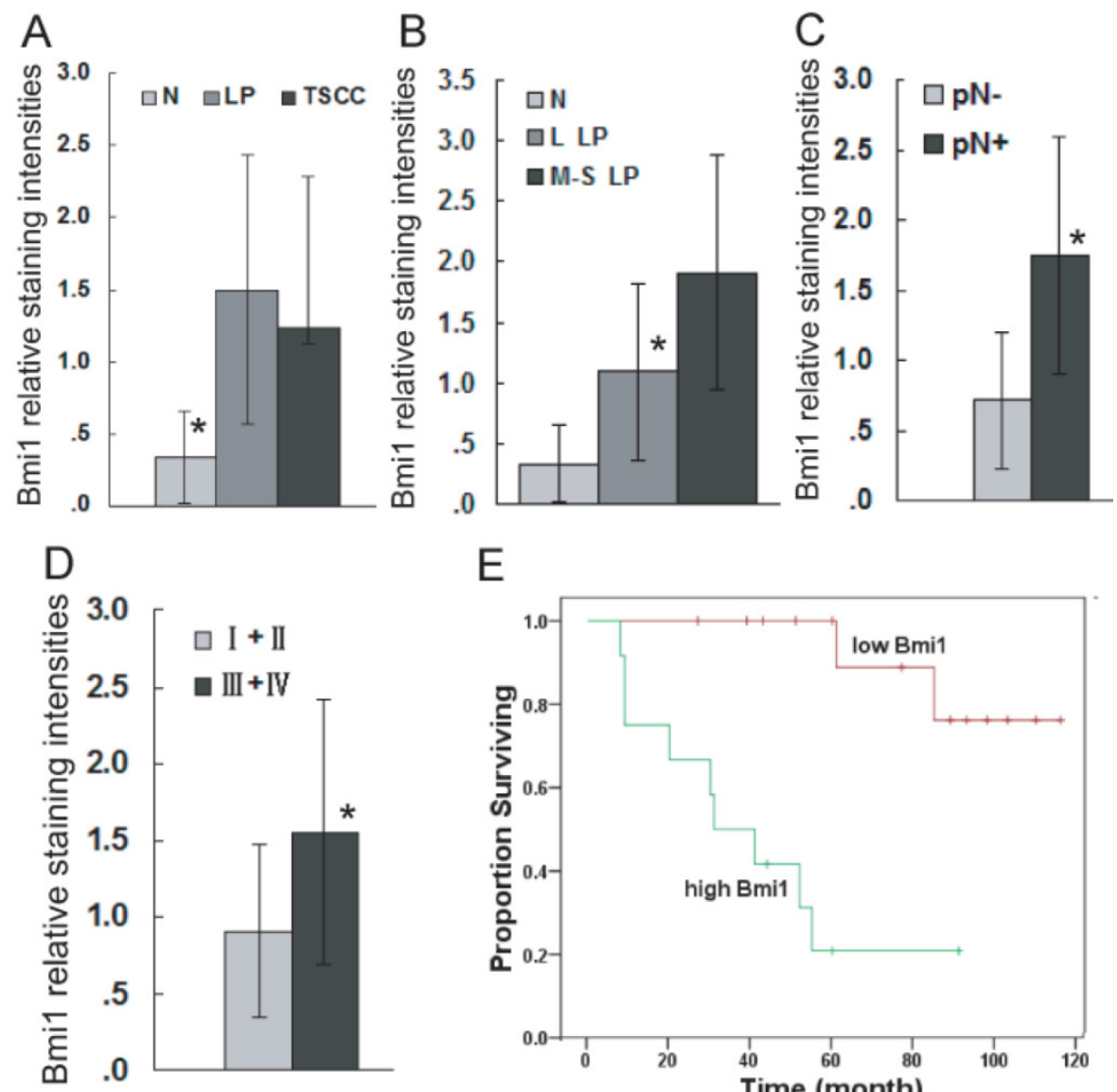

$\mathrm{E}$

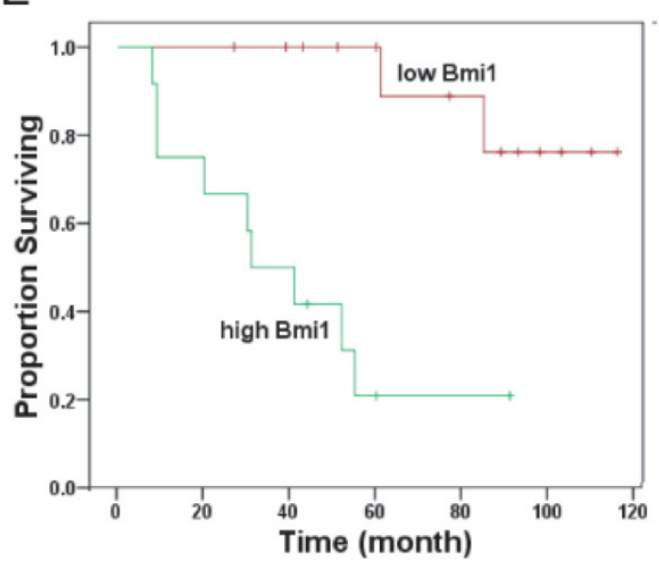

Figure 2. Bmil deregulation is associated with the development and poor prognosis of TSCC. Bmil staining intensities were presented for comparison among TSCC, LP, and normal tongue tissue (A: Bmil expression was significantly higher in LP and TSCC than in normal tongue tissue. No difference in the Bmil expression level was observed between TSCC and LP, TSCC and L LP, TSCC and M-S LP.), LP cases of different grades (B: Bmil expression was significant difference between normal and L LP, normal and M-S LP, L LP and M-S LP.), TSCC cases with or without lymph node metastasis (C), and TSCC cases of different clinical stages (D). Kaplan-Meier plots of overall survival in patient groups $(n=52)$ defined by Bmil immunohistochemistry was analyzed $(E)$. The differences in survival rates were statistically significant $(P<0.05)$. L LP: light LP, M-S LP: moderate and severe LP, High Bmil: Bmil expression higher than the mean staining in TSCC (1.26), Low Bmil: Bmil expressions lower than mean staining in TSCC (1.26). *P $<0.05$. The actual $P$ value for each of them was supplied in Supplementary Material: Table S3.

Table 1: Correlation of Bmil level with expression of its related genes in tumor tissues and clinical characteristics of TCSS patients.

\begin{tabular}{llllll}
\hline TSCC & & \multicolumn{3}{c}{ Pearson } & \\
\cline { 3 - 6 } & & r & P value & r & P value \\
\hline Bmi1 expression & Age & 0.127 & 0.246 & 0.148 & 0.175 \\
& Gender & -0.127 & 0.273 & -0.129 & 0.263 \\
& pT stage & -0.051 & 0.645 & -0.037 & 0.738 \\
& pN stage & 0.446 & 0.01 & 0.407 & 0.021 \\
& C stage & 0.356 & 0.004 & 0.343 & 0.006 \\
& SOD2 & 0.632 & 0.000 & 0.747 & 0.000 \\
& Ki67 & 0.453 & 0.000 & 0.468 & 0.000 \\
\hline
\end{tabular}

We further analyzed correlations among gene expression (e.g., Bmi1, SOD2, and Ki67) and clinical and pathological characteristics in the TSCC patient cohort (Table 1). The expression pattern of SOD2 and Ki67 had been published in our previous research [23]. There was no significant correlation between the expression of Bmi1 and age, gender, and pT stage in the present study. However, strong positive correlations were observed between $\mathrm{pN}$ and the clinical stage. A significant correlation was also observed between Bmi1 expression and SOD2 expression and the Ki67 proliferation index.

\section{Bmil-mediated TSCC migration and invasion is related to cancer stem-like cells and SOD2}

As shown in Figure 3A and S2A, Bmi1 has a higher expression level in UM1 cells than in UM2 cells. However, after the knockdown of Bmi1 in UM1 cells (Figure 3A), the migration (Figure 3B) and invasion (Figure $3 \mathrm{C}$ ) abilities were significantly inhibited compared to control siRNA transfection.

To investigate whether TSCC migration and invasion mediated by Bmi1 is related to cancer stem-like cells, we sorted and characterized SP cells (cancer stem-like cells) from TSCC cell lines. As shown in Supplementary Material: Figure S3, the proportion of SP cells, sorted by flow cytometry, was decreased in the presence of verapamil. The proportion of UM1 and UM2 SP cells was $3.2 \%$ and $2.5 \%$, respectively. The SP cells expressed a high level of Bmi1 and migration and invasion abilities in comparison to non-SP 
cells (Supplementary Material: Figure S4). As shown in Figure 3D, the proportion of SP cells was significantly decreased in UM1 cells after Bmi1 knockdown, and after 14 days of culture, the UM1 cells transfected with Bmil siRNA displayed significantly decreased sphere formation (Figure 3E) and colony formation (Figure 3F) compared to the cells transfected with control siRNA. The expression of SOD2, Slug, and stem cell markers (ABCG2, Nanog) in UM1 cells were significantly decreased after transfection with Bmi1 siRNA (Figure 3G and S2B). Moreover, the proliferation of UM1 cells was significantly inhibited after transfection with Bmil siRNA compared to the cells transfected with control siRNA (Figure 3H).

To further characterize the role of Bmi1 in the migration and invasion ability of cancer stem-like cells in TSCC, we knocked down the expression of Bmi1 (Figure 4A) in SP cells. As shown in Figure 4B-C, the SP cells transfected with Bmi1 siRNA displayed significantly decreased migration (Figure 4B) and invasion (Figure 4C) abilities compared to the control siRNA-transfected SP cells. Bmi1 knockdown resulted in significantly reduced sphere formation (Figure 4D), colony formation (Figure 4E), and proliferation (Figure $4 \mathrm{~F}$ ) in SP cells. We also found that the SP cells transfected with Bmi1 siRNA displayed significantly reduced expression levels of SOD2, Slug, and stem cell markers (ABCG2 and Nanog) (Figure 4A and S2C). Conversely, Bmi1 knockdown in UM1 and SP cells did not affect the expression of C-myc (Figure 3G, 4A, S2B and S2C). All of these data indicated that Bmi1-mediated TSCC migration and invasion is related to cancer stem-like cells. Furthermore, Bmi1 knockdown led to the down-regulation of SOD2 and Slug.
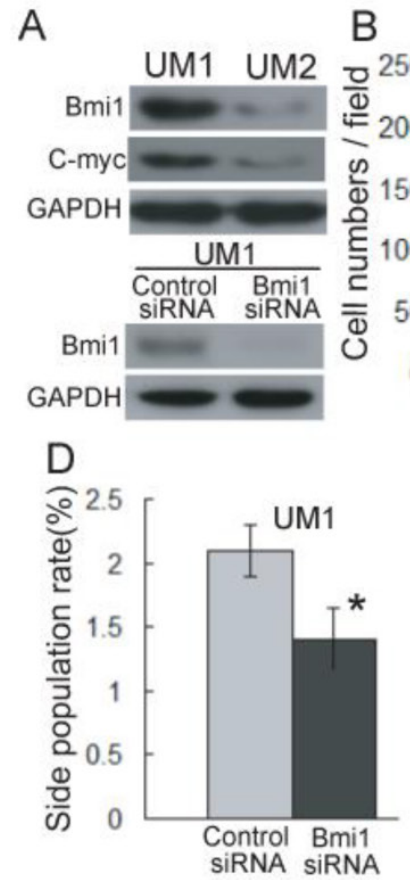
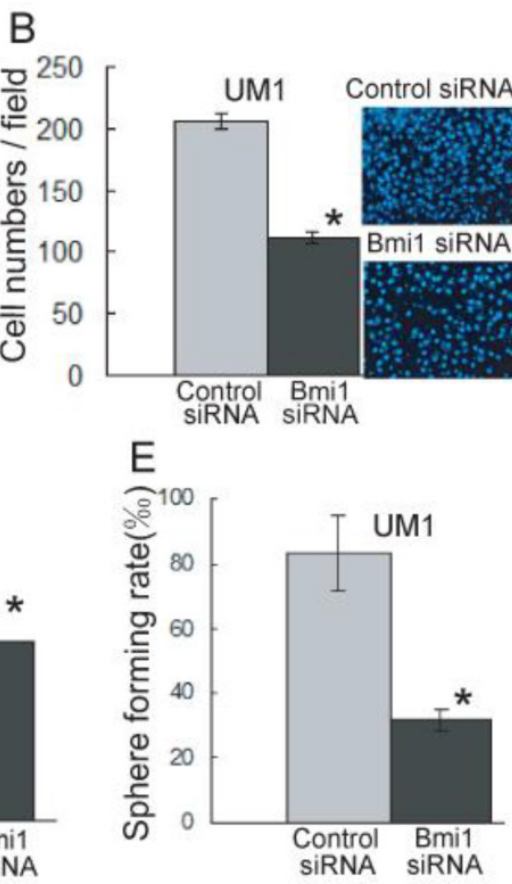
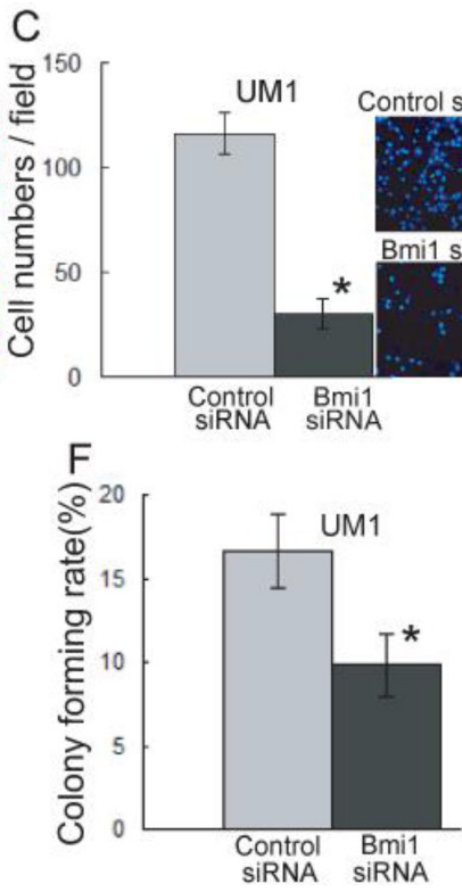

G
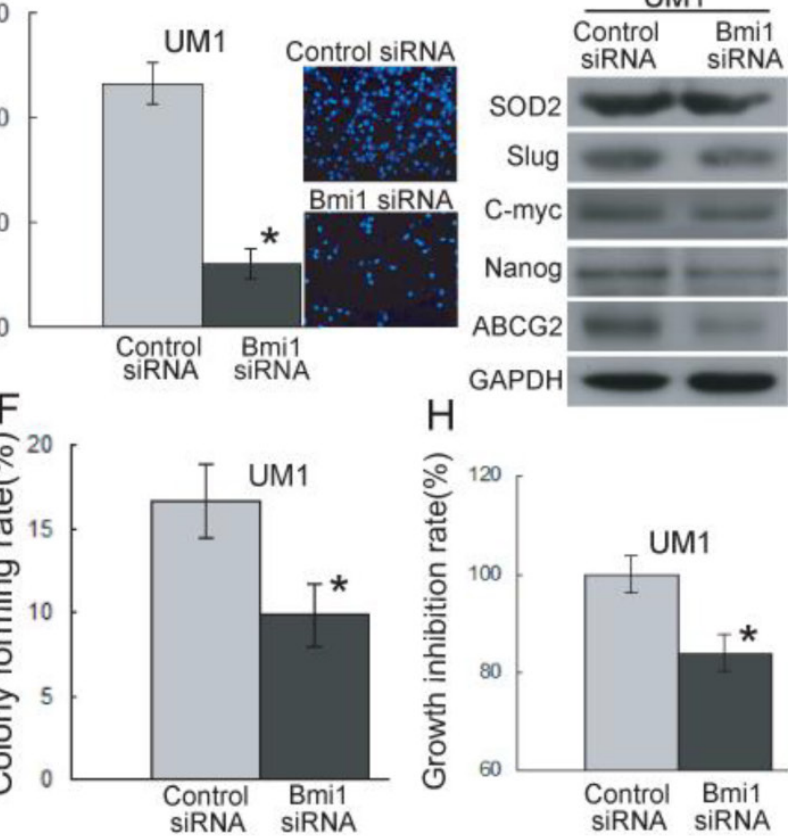

Figure 3. Bmil-mediated TSCC migration and invasion are related to cancer stem cells. (A) The expression of Bmil and C-myc was detected by western blotting using GAPDH as loading control. (B, C) The migration (B) and invasion (C) abilities in UMl cells after transfection with Bmil siRNA were significantly inhibited compared to control siRNA transfection. (D-F) Significantly higher SP subpopulation (D), sphere formation (E), and colony formation (F) were found in UMI cells transfected control siRNA compared to UM1 cells transfected with Bmil siRNA. (G) The expression of SOD2, Slug, and stem cell markers (ABCG2, Nanog) was decreased in UM1 cells transfected with Bmil siRNA. (H) UMl cells transfected with control siRNA showed a significantly higher proliferation rate than UMl cells transfected with Bmil siRNA. *P < 0.05 . 
A

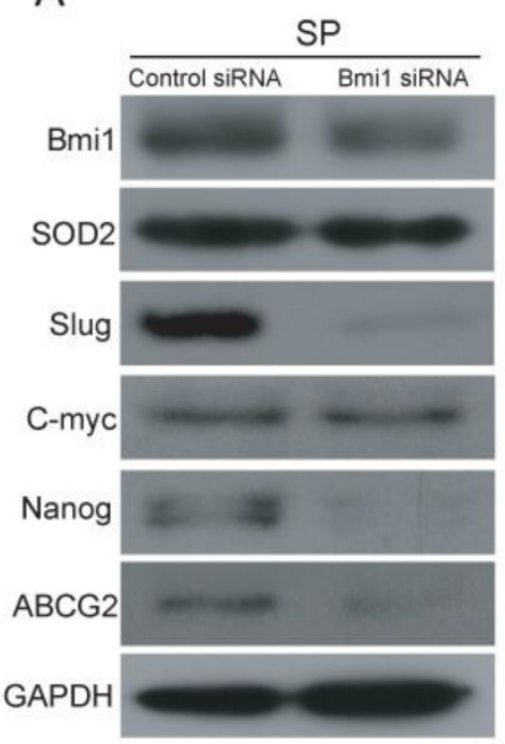

B
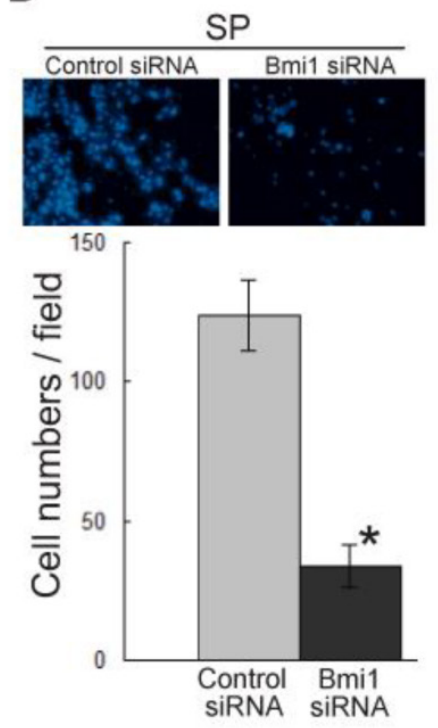

C
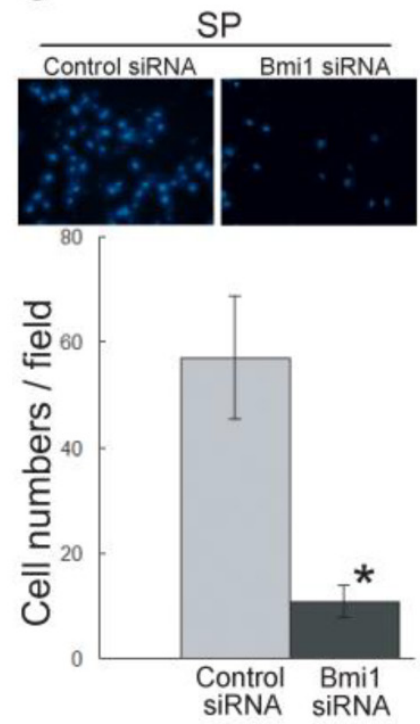
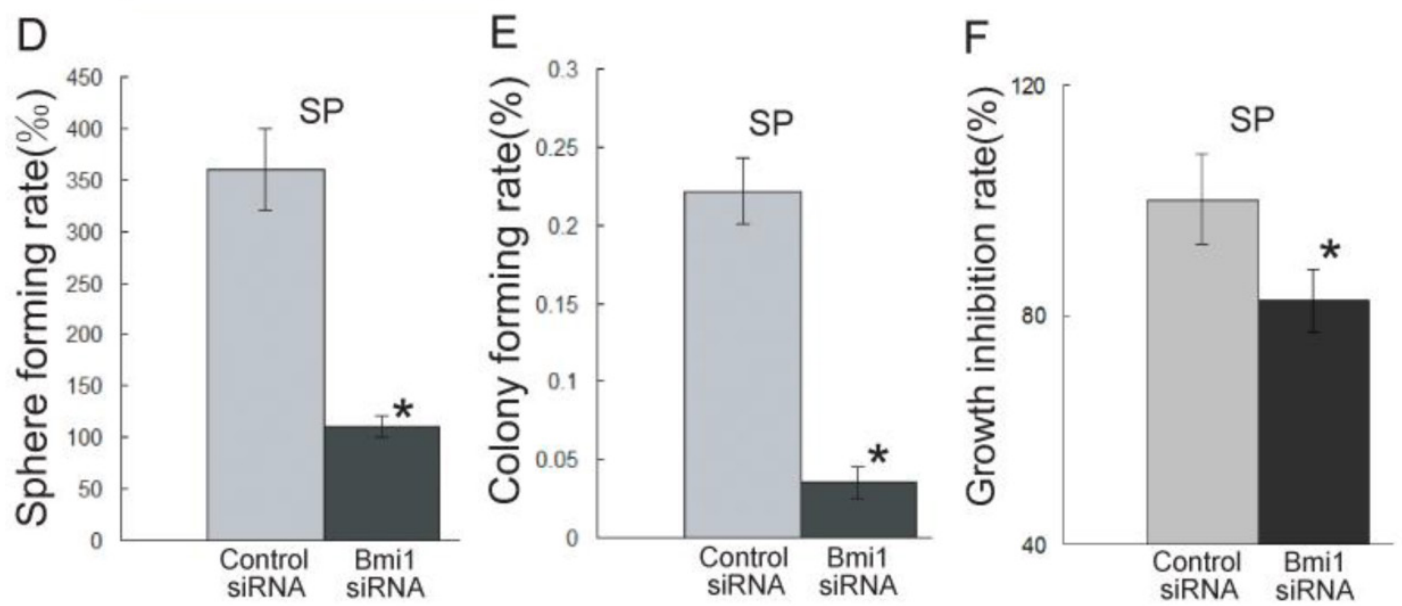

Figure 4. Bmil knockdown inhibits the migration and invasion ability of cancer stem-like cells (SP cells) in TSCC. (A) The expression of Bmil, SOD2, Slug, and stem cell markers (ABCG2, Nanog) was inhibited in SP cells transfected with Bmil siRNA. (B, C) The migration (B) and invasion (C) abilities were significantly inhibited in Bmil siRNA-transfected SP cells compared to control siRNA-transfected SP cells. (D-F) The SP cells transfected with Bmil siRNA had significantly decreased sphere formation (D, $\mathrm{P}=0.0005)$, colony formation $(\mathrm{E}, \mathrm{P}=0.0002)$, and proliferation $(\mathrm{F}, \mathrm{P}=0.001)$ compared to control siRNA-transfected $\mathrm{SP}$ cells. $* \mathrm{P}<0.05$.

\section{C-myc directly targets the Bmil-mediated migration and invasion of cancer stem-like cells in TSCC}

As shown in Figure 5A, a C-myc recognition site is present in the promoter of Bmi1 genes (http://genome.ucsc.edu/), suggesting that the expression of Bmi1 may be regulated by C-myc. To address whether $\mathrm{C}$-myc directly binds to the promoter of Bmi1 in TSCC cells, ChIP assays were performed. As shown in Figure 5B and Figure 5C, a significantly higher level of C-myc binding to the promoter of Bmi1 was shown for the UM1 cells versus the UM2 cells. To further investigate whether Bmi1 is transcriptionally induced by C-myc, a dual luciferase assay was performed. UM2 cells expressing a C-myc expression vector, co-transfected with pGL-Bmil, exhibited significantly increased Bmi1 promoter activity compared with UM2 cells expressing the control vector (Figure 5D). The luciferase activity in UM2 cells transfected with pGL-Bmi1mt did not respond to induction by C-myc (Figure 5D). Subsequently, we analyzed the regulation of Bmi1 by $\mathrm{C}$-myc by knocking down C-myc expression in SP cells, which show a high expression of C-myc than non-SP cells (Figure S4). As expected, the expression of Bmi1 was inhibited in SP cells after transfection with C-myc siRNA (Figure 5E and S2D); the SP cells transfected with C-myc siRNA also displayed decreased migration and invasion abilities compared to the control siRNA-transfected cells (Figure 5F and 5G). Furthermore, SP cells transfected with C-myc siRNA displayed reduced SOD2 expression (Figure 5E and S2D). These results indicated C-myc directly regulates Bmi1 and mediates the migration and invasion of cancer stem-like cells in TSCC. 
A

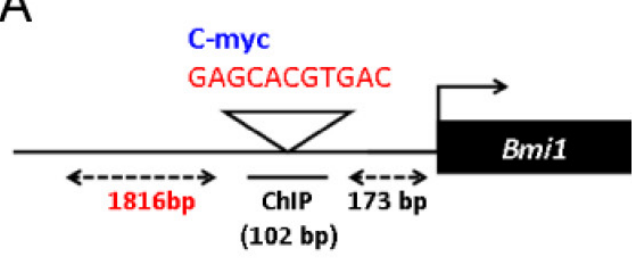

B

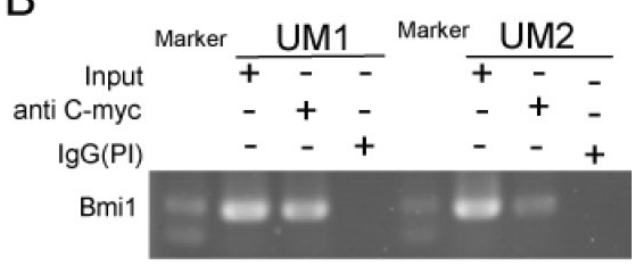

E

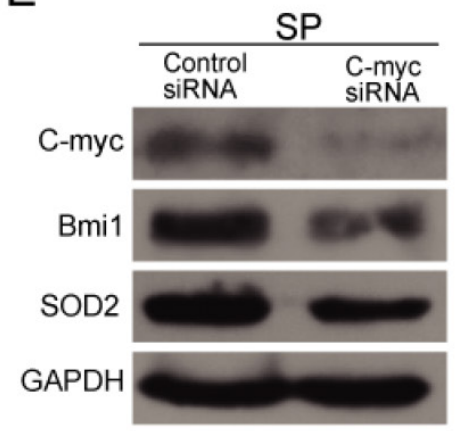

C

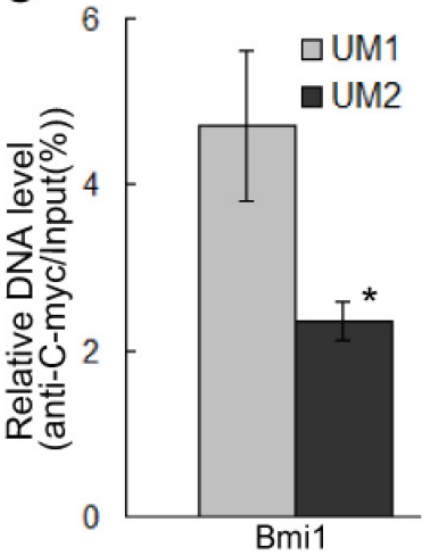

$\mathrm{F}$

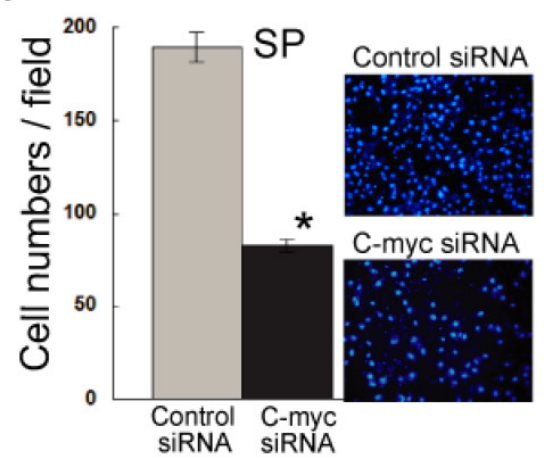

D

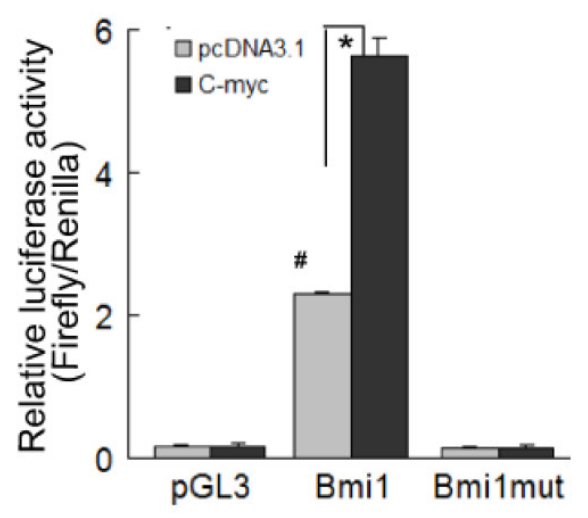

G

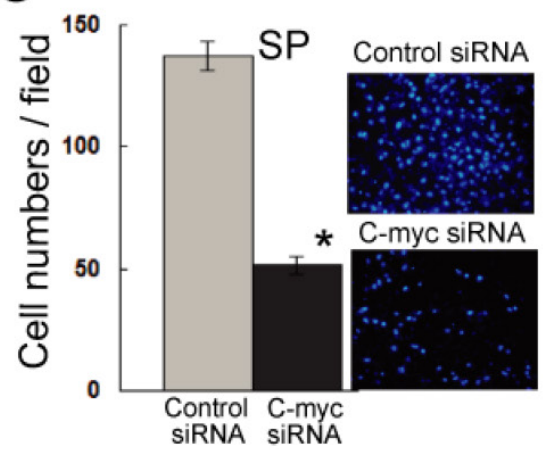

Figure 5. C-myc directly targets Bmil and mediates the migration and invasion of SP cells in TSCC. (A) The predicted C-myc target sites in the promoter of Bmil (NM 005180) are presented. (B, C) Endogenous C-myc binding to the Bmil promoter was assessed by a CHIP assay. Rabbit IgG was used as the negative control (PI). Quantitative PCR (C) was carried out on immunoprecipitated chromatin (IP by C-myc) and input chromatin and expressed as $2 \mathrm{Ct}_{\text {input }}{ }^{-} \mathrm{Ct}$ IP $\times 100 \%$. (D) Bmil promoter activity induced by $\mathrm{C}$-myc was detected by a luciferase assay. UM2 cells were transiently cotransfected with pGL-Bmil or pGL-Bmil-mt with an expression construct for C-myc or an empty vector (\#: compared with pcDNA3.1, P < 0.05; this may be related to the low level expression of C-myc in UM2 cells.). (E) The expression of C-myc, Bmil, and SOD2 was inhibited in SP cells transfected with C-myc siRNA. (F, G) The migration (F) and invasion (G) abilities were significantly inhibited in C-myc siRNA-transfected SP cells compared to control siRNA-transfected SP cells. $* \mathrm{P}<0.05$.

\section{Discussion}

Bmi-1 is a stem cell marker associated with head and neck tumorigenesis. Studies have revealed that Bmi-1 expression is associated with the development of oral cancer in patients with oral leukoplakia (LP) [14], oral erythroplakia [11], and oral lichen planus [15]. In clinical specimens of HNSCC, many researchers have found that the overexpression of Bmi-1 correlates with poor overall survival $[13,16,31]$. Similarly, we also found that the expression of Bmi1 was higher in TSCC and correlated with a poor overall survival and was also associated with lymph node metastasis and clinical stage. Moreover, Bmi-1 overexpression was found in LP, with a significant difference between light LP and moderate and severe LP. Consistent results were reported in Kang`s research [32]. These results indicate that Bmi-1 expression may occur at a very early stage in tongue carcinogenesis and that Bmi1 is an important factor in the development and progression of TSCC. Conversely, a statistically significant correlation was in reported by Hayry et al between lack of Bmi-1 immunoexpression and a poor prognosis in TSCC patients [17]. The apparent difference may be explained by the fact that our TSCC patient cohort included all staged of TSCC, whereas that of Hayry et al only included pT1 and pT2 [17].

Robust evidence suggests that Bmi1 is critical to invasive potential and contributes to the maintenance and self-renewal of CSCs in several tumor types, including HNSCC [7, 13, 33, 34]. For example, Chou`s study found that Bmi1 overexpression drives stem-like properties associated with invasion, metastasis, and poor prognosis in HNSCC [13]. As HNSCC includes a group of diverse cancers that develop from many different anatomic sites and is associated with different risk factors and genetic characteristics [35], it is necessary to clarify signature gene sets for tumor metastases of HNSCC originating from different anatomic sites. To date, few studies have focused on the relationship between Bmi1 and the metastasis of TSCC. In our study, we found that Bmi1 was overexpressed in UM1 cells and SP cells, both with higher migration and invasion abilities. The knockdown of Bmi1 in UM1 cells or SP cells inhibited cell prolifera- 
tion and in vitro migration and invasion and decreased sphere formation, clone formation, and the expression of stem cell markers (Nanog and ABCG2, which had been found to be cancer stem cell markers of TSCC [36,37]). SP cells from TSCC were found to possess the characteristics of cancer stem-like cells, possessing a higher proportion of sphere and colony formation and a higher expression of stem cell markers. These results indicate that the Bmi1-mediated migration and invasion of TSCC is related to cancer stem-like cells.

Our previous studies had found that the SOD2-dependent production of $\mathrm{H}_{2} \mathrm{O}_{2}$ contributes to the migration and invasion abilities of TSCC via the Snail signaling pathway [22-24]. Many studies have reported that Snail promotes invasion in many types of cancers $[16,28,38]$, including salivary adenoid cystic carcinoma [28] and TSCC [38]. Yu`s study suggested that Bmi-1 plays a key role in regulating Snail expression and the cancer stemness properties of HNSCC-ALDH1(+) cells [16]. However, the relationship between Bmi1 and the SOD2-Slug pathway in TSCC has not been reported. In this study, we found that the expression of Bmi1 was correlated with SOD2 in TSCC tissues. Bmi1 knockdown inhibited the expression of SOD2 and Slug in UM1 and SP cells. $\mathrm{C}$-myc is a transcription factor that recognizes the E-box sequence and several related noncanonical sequences in target genes $[19,39,40]$, and recent studies showed that Bmi1 is a direct transcriptional target of $\mathrm{C}$-myc $[8,21]$. In the present study, we also verified that C-myc binds to the E-box sequence in the promoter of Bmi1. As the knockdown of C-myc inhibited the expression of Bmi1 and SOD2 and the migration and invasion of SP cells, our results indicate that $\mathrm{C}$-myc directly targets $\mathrm{Bmi1}$ and that Bmi1 regulates the SOD2-Slug pathway in TSCC.

Altogether, the present findings reveal that Bmi1 plays an important role in the development of TSCC. The Bmi1-mediated migration and invasion of TSCC is related to cancer stem-like cells. C-myc directly targets and regulates the expression of Bmil. Moreover, Bmi1 regulates the SOD2-Slug pathway and mediates the migration and invasion of TSCC.

\section{Abbreviations}

Bmi1: B-cell-specific Moloney murine leukemia virus insertion site 1; SOD2: manganese superoxide dismutase; TSCC: tongue squamous cell carcinoma; LP: leukoplakia; OSCC: oral squamous cell carcinoma; HNSCC: head and neck cancer; RNA: Ribonucleic Acid; CSCs: cancer stem cells; SP: side population; IHC: immunohistochemistry; ChIP: Chromatin immunoprecipitation; PCR: polymerase chain reaction.

\section{Supplementary Material}

Tables S1-S3, Figures S1 - S4.

http://www.ijbs.com/v11p0001s1.pdf

\section{Acknowledgments}

This work was supported, in part, by grants from the National Nature Science Foundation of China (NSFC81472523, NSFC81272953), the Guangdong Natural Science Foundation (S2011020002325), the research fund for the doctoral program of Ministry of Education (20120171110050), the fundamental research funds for the Central Universities (11ykzd09), and the program for New Century Excellent Talents in University (NCET-10-0857).

\section{Conflict of Interest}

The authors have declared that no competing interest exists.

\section{References}

1. Haddad RI, Shin DM. Recent advances in head and neck cancer. N Engl J Med. 2008;359:1143-54.

2. Zou B, Sun S, Qi X, et al. Aldehyde dehydrogenase activity is a cancer stem cell marker of tongue squamous cell carcinoma. Mol Med Rep. 2012;5:1116-20.

3. Rastogi P. Emergence of cancer stem cells in head and neck squamous cell carcinoma: A therapeutic insight with literature review. Dent Res J (Isfahan). 2012;9:239-44.

4. Ailles L, Prince M. Cancer stem cells in head and neck squamous cell carcinoma. Methods Mol Biol. 2009;568:175-93.

5. Prince ME, Sivanandan R, Kaczorowski A, et al. Identification of a subpopulation of cells with cancer stem cell properties in head and neck squamous cell carcinoma. Proc Natl Acad Sci USA. 2007;104:973-8.

6. Chinn SB, Darr OA, Peters RD, et al. The role of head and neck squamous cell carcinoma cancer stem cells in tumorigenesis, metastasis, and treatment failure. Front Endocrinol (Lausanne). 2012; 3:90.

7. Proctor E, Waghray M, Lee CJ, et al. Bmi1 enhances tumorigenicity and cancer stem cell function in pancreatic adenocarcinoma. PLoS One. 2013;8:e55820.

8. Huang R, Cheung NK, Vider J, et al. MYCN and MYC regulate tumor proliferation and tumorigenesis directly through BMI1 in human neuroblastomas. Faseb J. 2011;25:4138-49.

9. Tsai LL, Yu CC, Chang YC, et al. Markedly increased Oct4 and Nanog expression correlates with cisplatin resistance in oral squamous cell carcinoma. J Oral Pathol Med. 2011;40:621-8.

10. Seo E, Basu-Roy U, Zavadil J, et al. Distinct functions of Sox2 control self-renewal and differentiation in the osteoblast lineage. Mol Cell Biol. 2011;31:4593-608.

11. Feng JQ, Xu ZY, Shi LJ, et al. Expression of cancer stem cell markers ALDH1 and Bmi1 in oral erythroplakia and the risk of oral cancer. J Oral Pathol Med. 2013;42:148-53.

12. Li Z, Wang Y, Yuan C, et al. Oncogenic roles of Bmi1 and its therapeutic inhibition by histone deacetylase inhibitor in tongue cancer. Lab Invest. 2014; doi: 10.1038/labinvest.

13. Chou CH, Yang NK, Liu TY, et al. Chromosome instability modulated by BMI1-AURKA signaling drives progression in head and neck cancer. Cancer Res. 2013;73:953-66.

14. Liu W, Feng JQ, Shen XM, et al. Two stem cell markers, ATP-binding cassette, G2 subfamily (ABCG2) and BMI-1, predict the transformation of oral leukoplakia to cancer: a long-term follow-up study. Cancer. 2012;118:1693-700

15. Ma L, Wang $\mathrm{H}$, Yao $\mathrm{H}$, et al. Bmi1 expression in oral lichen planus and the risk of progression to oral squamous cell carcinoma. Ann Diagn Pathol. 2013:17:327-30.

16. Yu CC, Lo WL, Chen YW, et al. Bmi-1 Regulates Snail Expression and Promotes Metastasis Ability in Head and Neck Squamous Cancer-Derived ALDH1 Positive Cells. J Oncol. 2011; doi: 10.1155/2011/609259.

17. Hayry V, Makinen LK, Atula T, et al. Bmi-1 expression predicts prognosis in squamous cell carcinoma of the tongue. Br J Cancer. 2010;102:892-7.

18. den Hollander J, Rimpi S, Doherty JR, et al. Aurora kinases A and B are up-regulated by Myc and are essential for maintenance of the malignant state. Blood. 2010;116:1498-505.

19. Ozawa N, Shichiri M, Fukai N, et al. Regulation of adrenomedullin gene transcription and degradation by the c-myc gene. Endocrinology. 2004;145:4244-50. 
20. Bretones G, Acosta JC, Caraballo JM, et al. SKP2 oncogene is a direct MYC target gene and MYC down-regulates p27(KIP1) through SKP2 in human leukemia cells. J Biol Chem. 2011;286:9815-25.

21. Wang $\mathrm{HB}$, Liu GH, Zhang $\mathrm{H}$, et al. $\mathrm{Sp} 1$ and c-Myc regulate transcription of BMI1 in nasopharyngeal carcinoma. Febs J. 2013;280:2929-44.

22. Ye H, Wang A, Lee BS, et al. Proteomic based identification of manganese superoxide dismutase 2 (SOD2) as a metastasis marker for oral squamous cell carcinoma. Cancer Genomics Proteomics. 2008;5:85-94.

23. Liu X, Wang A, Lo Muzio L, et al. Deregulation of manganese superoxide dismutase (SOD2) expression and lymph node metastasis in tongue squamous cell carcinoma. BMC Cancer. 2010;10:365.

24. Liu Z, Li S, Cai Y, et al. Manganese superoxide dismutase induces migration and invasion of tongue squamous cell carcinoma via H2O2-dependent Snail signaling. Free Radic Biol Med. 2012;53:44-50.

25. Ding X, Zhang N, Cai Y, et al. Down-regulation of tumor suppressor MTUS1/ATIP is associated with enhanced proliferation, poor differentiation and poor prognosis in oral tongue squamous cell carcinoma. Mol Oncol. 2012;6:73-80.

26. Wang A, Liu X, Sheng S, et al. Dysregulation of heat shock protein 27 expression in oral tongue squamous cell carcinoma. BMC Cancer. 2009;9:167.

27. Li J, Gong LY, Song LB, et al. Oncoprotein Bmi-1 renders apoptotic resistance to glioma cells through activation of the IKK-nuclear factor-kappaB Pathway. Am J Pathol. 2010;176:699-709.

28. He O, Zhou X, Li S, et al. MicroRNA-181a suppresses salivary adenoid cystic carcinoma metastasis by targeting MAPK-Snai2 pathway. Biochim Biophys Acta. 2013;1830:5258-66.

29. Yao CJ, Lai GM, Yeh CT, et al. Honokiol Eliminates Human Oral Cancer Stem-Like Cells Accompanied with Suppression of Wnt/ beta -Catenin Signaling and Apoptosis Induction. Evid Based Complement Alternat Med. 2013;2013:146136.

30. Wang A, Alimova IN, Luo P, et al. Loss of CAK phosphorylation of RAR\{alpha\} mediates transcriptional control of retinoid-induced cancer cell differentiation. Faseb J. 2010;24:833-43.

31. Chen H, Zhou L, Wan G, et al. BMI1 promotes the progression of laryngeal squamous cell carcinoma. Oral Oncol. 2011;47:472-81.

32. Kang MK, Kim RH, Kim SJ, et al. Elevated Bmi-1 expression is associated with dysplastic cell transformation during oral carcinogenesis and is required for cancer cell replication and survival. Br J Cancer. 2007;96:126-33.

33. Major AG, Pitty LP, Farah CS. Cancer stem cell markers in head and neck squamous cell carcinoma. Stem Cells Int. 2013;2013:319489.

34. Chen YC, Chang CJ, Hsu HS, et al. Inhibition of tumorigenicity and enhancement of radiochemosensitivity in head and neck squamous cell cancer-derived ALDH1-positive cells by knockdown of Bmi-1. Oral Oncol. 2010;46:158-65.

35. Zhou X, Temam S, Oh M, et al. Global expression-based classification of lymph node metastasis and extracapsular spread of oral tongue squamous cell carcinoma. Neoplasia. 2006;8:925-32.

36. Yanamoto S, Kawasaki G, Yamada S, et al. Isolation and characterization of cancer stem-like side population cells in human oral cancer cells. Oral Oncol. 2011;47:855-60.

37. Lim W, Choi H, Kim J, et al. Expression of cancer stem cell marker during 4-nitroquinoline 1-oxide-induced rat tongue carcinogenesis. J Mol Histol. 2014; doi 10.1007/s10735-014-95 84-1

38. Wang C, Liu X, Huang H, et al. Deregulation of Snai2 is associated with metastasis and poor prognosis in tongue squamous cell carcinoma. Int J Cancer. 2012;130:2249-58.

39. Fujioka Y, Taira T, Maeda Y, et al. MM-1, a c-Myc-binding protein, is a candidate for a tumor suppressor in leukemia/lymphoma and tongue cancer. J Biol Chem. 2001;276:45137-44.

40. Guney I, Wu S, Sedivy JM. Reduced c-Myc signaling triggers telomere-independent senescence by regulating Bmi-1 and p16(INK4a). Proc Natl Acad Sci USA. 2006;103:3645-50. 